\title{
IMAGE CAPTURE FOR CONCRETE PROGRAMMING Building Schemata for Problem Solving
}

\author{
Vladimir Estivill-Castro and Brendan Bartlett \\ Griffith University, Brisbane, Australia
}

Keywords: Information technologies supporting learning, Software tools.

\begin{abstract}
Problem solving in IT consists of expressing an algorithm for abstract models of computation. This has proven to be hard, but it can be taught, specially when students are exposed to concrete and visual illustrations of artefact behaviour. IT graduates require problem-solving skills, but it is difficult to teach such problem-solving skills in the context of huge bodies of technological concepts, large programming languages and the need for system-focused courses required for accreditation. We propose to design and develop concrete programming activities that will enable to articulate problem solving across many subjects. The goal is also to place concepts in the context of concrete problems, and to progress from concrete settings (where programming is achieved by building structures) to visual settings (where programming is achieved by re-arranging icons in a GUI), and later to textual programming in imperative APIs like MaSH. We capture the participants constructions with a camera and this is a program that produces behavior. The approach delivers the potential to take students to investigate research questions.
\end{abstract}

\section{INTRODUCTION}

Students graduating from Information and Communication Technology degrees should be able to perform problem solving by expressing the solution algorithmically. The ability to solve problems with a degree of creativity is highlighted as an essential characteristic for both novice undergraduate engineers and qualified IT professionals in benchmarks published by the OECD (Houghton, 2004). Problem solving is crucial to the professional success of graduates of the School of Information and Communication Technology at Griffith University, Australia. It is usually manifested by the ability to program a computer so that, from any set of input values that constitute a valid instance of the problem, the computer finds out the solution to such instance. In fact, such abilities are considered relevant even from high-school. Under the label of "Working ways" high-school students in Queensland are expected to

- plan activities and investigations to explore concepts,

- plan strategies to solve mathematical questions, problems and issues,

- evaluate thinking and reasoning,
- perform thinking and working and reasoning that can be applied to solve problems in real-life and abstract investigations.

Problem solving for IT graduates means not only solving problems (Dewar, 2006), but also expressing a method, an algorithm, in the language that defines the operation of an automaton (Dale and Weems, 2002). This means not only figuring out the answer to a problem, but also describing the step-by-step process that a machine (with no intelligence or common sense) would be performing to obtain the answer. In this scenario, there is much creativity, analysis and design skills in developing solutions, since there is usually a large body of acceptable solutions.

Algorithmic problem-solving thinking is at the foundation of rational thinking in our civilization. Greek thinkers where particularly interested in constructive methods as illustrated by Euclid's elements. We argue here that such approach can be regarded as concrete programming. It offered a few basic operations (usually to draw a circle with a compass and to draw a straight line with a ruler). Propositions and their proofs were algorithms and proofs of correctness for such algorithms (Toussaint, 1993a; Toussaint, 1993b). The model of computation had a physical analogue and it was possible to experiment with 
a compass and a ruler on an instance of the problem.

We suggest that this approach should be used to introduce students to programming. Our approach is in contrast to the tradition to link closely the instruction on problem solving to instruction in syntax and semantics of programming languages (Schneider, 1982; Koffman, 1988; Etter and Ingber, 1999; Savitch, 2009). We question and seek an earlier connection between problem solving and computer programming. We propose to move gradually from concrete personal experiences to symbolic textual (abstract) programming. We propose that the technology is already there for capturing (in images and video) the concrete constructions by the students, and that such constructions should represent behaviour.

We aim for student involvement in action and aim for better outcomes of first year IT students. The prompt for this improvement will be a program of staged activities through which students build their skills and agency in problem solving at both a metalevel and for specific skills they will need in the first year course-work. We describe one developmental activity with an emphasis on concrete operations moving through to an informed and abstracted familiarity. We aim to strengthen students' building schemata by associating their action with appropriate language for its labelling and discussion.

Problem-solving skills are more important than learning several programming languages, because the technologies to program computers vary constantly - computers are faster and with more memory, but they remain fundamentally state-transition machines. Thus, learning a particular programming language is ephemeral; describing solutions to problems algorithmically is and will continue to be an endurable skill. However, learning the problem-solving skills necessary for programming has proven to be hard, and so has teaching them (Adams and Turner, 2008). It requires significant conceptual and abstract thinking, regularly associated with the skills for solving mathematical problems (Polya, 1957). However, for IT students, there are additional challenges.

First Challenge: the generation of an algorithm that works for all instances of the problem. Issues are complicated because is impossible to perceive with our senses what goes on on a computer (let alone through entities like the Internet and the Word Wide Web). A large literature on teaching problem solving and a discussion on analytical skills in physics, mathematics and engineering is presented by James E. Stice at University of Texas ${ }^{1}$ mostly deriving from the pioneering

\footnotetext{
${ }_{1}$ wwwcsi.unian.it/educa/problemsolving/stice_ps.html
}

work of Donald R. Woods (Woods et al., 1975). Other literature and its references cover many aspects of teaching problem solving (Adams et al., 2007; Prince and Hoyt, 2002). For IT, teaching and learning problem solving has the added complexity of explaining and coding the solution in a programming language.

Second Challenge: the abstraction of the description; namely, the language in which the solution is expressed is an abstraction of the changes of state that occur in abstractly described objects. The complications of programming computers have resulted in the software crisis. Producing software is extremely difficult, and usually does not meet user expectations, it is faulty and needs upgrades. The price of more powerful hardware drops while inferior software remains costly. New software developing tools are more sophisticated and powerful. But such advances require more concepts, from structured programming, to object-oriented and recently to agent programming, for example. Moreover, with each of these advancements, the teaching of problem solving competes with the need to train the IT-students in sophisticated environments as well as the need to introduce them to a large body of concepts. As a result, some students perceive fundamental concepts as unrelated and even irrelevant. However, the relevant knowledge and common thread is indeed problem solving and techniques to obtain algorithms.

\section{THE STRATEGY}

Our first element is to develop learning activities performed by students in teams. These activities must be performed outside computer labs and must produce concrete constructions. Participants collaborate in teams, around their constructions and not in the difficult setting of sharing one monitor or a keyboard. We remove the arrangement of participants facing in one direction, to participants in collaborative arrangements surrounding a construction that is captured and interpreted by digital media. Then the software in computer vision and image analysis is used to convert the construction into meaningful behaviour in an automaton. Activities ${ }^{2}$ relate to knowledge introduced in at least 6 but possibly all 8 first-year courses of the Bachelor of IT. While such knowledge is not a prerequisite, the activities introduce it, reinforce it, or illustrate it by providing additional context. The activities integrate concepts so students can establish re-

\footnotetext{
${ }^{2}$ Activities are independent, but thematically linked.
} 


\section{GLOBAL STEPS: \\ 1. UNDERSTAND THE PROBLEM \\ 2. DEVISE A PLAN \\ 3. CARRY OUT THE PLAN \\ 4. LOOK BACK AND REFLECT \\ 1. Examine the solution obtained. \\ 2. Can you check the result? Can you check the argument? \\ 3. Can you derive the solution differently? Can you see it at a glance? \\ 4. Can you use the result, or the method, for some other problem?}

Figure 1: Basic/classical approach to problem solving.

lationships between courses. For example, activities on graph algorithms will incorporate concepts like accumulators, counters and absolute addressing from 1007ICT Introduction to Computer Systems and Networks but also notions like graphs from 1002ICT Discrete Structures 1. Activities whose foundations are state-automata, logic gates and Turing machines will be linking with 1004ICT Foundations of Computing and Communication and 1007ICT again. Complexity of software development and problem solving will take from 1410ICT Introduction to Information Systems and 1420ICT Systems Analysis and Design. Problem solving for programming is taken from 1001ICT Programming 1 and 1005ICT Programming 2. Activities will also be monitored across vertical paths of the program.

The second element of the strategy is that activities can be extra curricular and students participate in them by invitation and voluntary. Activities can be presented within the framework of a competition (in a similar fashion to the Science an Engineering Challenge) with interesting prizes (for added motivation). Activities can also be adopted by course conveners. For example, some of the activities may become required assessment in some of the courses mentioned earlier; however, this would be if adopted by lecturers or conveners of some of the courses.

Educational theories like Constructive Alignment (Biggs, 1999) suggest students should be aware that the learning outcomes are problemsolving skills and we will follow this suggestion. Therefore, the next element of the strategy is a guide on Teaching Problem Solving based on the theory from technical fields like engineering and mathematics (Wickelgren, 1995; Houghton, 2004) (and the Higher education Academy Engineering Subject Center on Problem Solving [www.engsc.ac.uk/er/theory/problemsolving.asp]

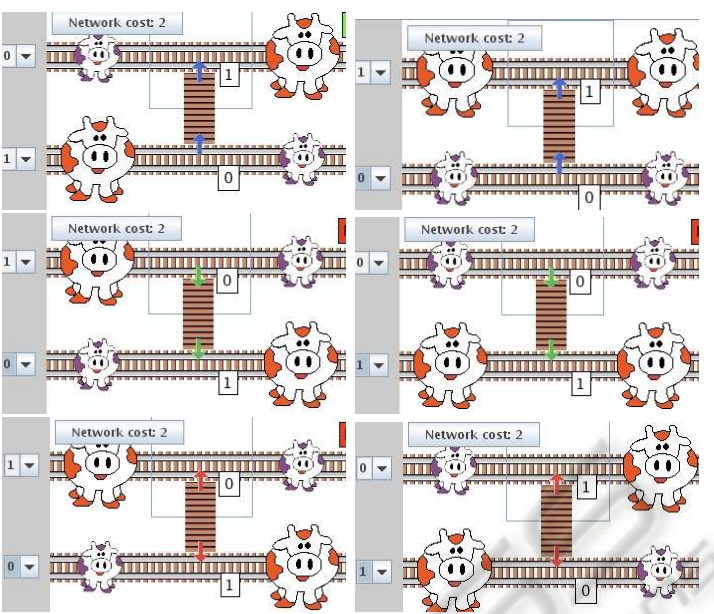

Figure 2: The basic operations.

and its corresponding supporting educational theories (Houghton, 2004)) but adapted to information technology and problem solving for algorithm development. We will use Constructive Alignment (Biggs, 1999) in order to ensure our assessment methods and our learning activities achieve the intended learning outcomes; namely improved problem-solving skills. Our intention is to evaluate under the following definition "Problem solving is the process of obtaining a satisfactory solution to a novel problem, or at least a problem which the problem solver has not seen before" (Woods et al., 1975). We may extend this to problem solving for software development and systems architecture.

A fundamental difficulty in teaching ICT at Griffith University is the limited ability of a substantial percentage of the student cohort to think in abstract terms (we explained before the low OP scores achieved by students enrolling in the programs (Estivill-Castro, 2010)). This restricts the student's ability to solve problems, as well as their ability to recognize the potential of applying already known knowledge in unfamiliar new situations (which, if described in abstract terms, proves to be the same or similar to an already known problem). The planned teaching / learning methodology we apply has some distinct characteristics:

- In the activities, students are presented with concrete problems and participate in solving them. But the activities engage students with different levels of abstract thinking ability to successfully participate.

- Through a series of increasingly difficult exercises involving guidance (cf. Social Constructivism), answering 'what if' (Brown and Walter, 1990; Brown and Walter, 1970) questions. With inde- 
pendent discovery, students construct and reflectively explain a generic solution, which leads to an implementation / algorithm - this approach not only ensures to test whether students managed to constructed their own understanding, but also develops their abstraction skills.

- Exercises exploring the limits of the solution (through comparing the problem to other similar ones and through asking 'what if not' questions) will help the learner to develop skills to recognize concrete situations in which known abstract solutions apply.

The constructive approach and the use of teachback (Pask, 1975; Vygotsky, 1978), ensure that the understanding of the problem and the understanding of the solution become an observable occasion. Our design of activities (to be performed by teams of students) is around a series of puzzles for realistic problems. In these problems, the students will construct a solution, but instead of initially using a keyboard/computer (and a formal programming language as in traditional text-based programming), they will commence by constructing physical artefacts. We refer to this as concrete programming although it has similarities with puzzle-based learning (Michalewicz and Michalewicz, 2008) and with problem-based learning (Cindy E. Hmelo-Silver et al., 2007, and references). We demonstrate here that high-school students can participate in the activities including some instruction in textual programming.

Our approach is to use concrete physical objects to build first phase solutions: here, the students will have playing cards, or other physical components (like LEGO or Konex) and they will build concrete physical solutions (but still descriptions of algorithms) to small instances of the puzzles. The students will be asked to attempt to obtain algorithms that work in general. However, these algorithms may fail in some of the many configurations. These will incorporate elements of role-play in learning. The second component of our approach is to use automated assessment: we propose to use a digital camera to capture the constructions (algorithms) of the students, and develop image processing software to interpret their physical programs; then execute them (run them) with all possible inputs and award them a score relative to the fraction of inputs in which they are correct. The third phase of our approach is programming by simulation.

The approach is close to visual programming, but tackling larger problems than with the physical manipulation of 'LEGO-brick' or 'playing cards'. However, we developed complementary educational software that emulates the puzzles and the concrete pro- gramming languages. Participants use simulations of cards, and tiles, and compose programs in the same way as in the physical world, but all in a Graphical User Interface (GUI). For example, RoboLab by LEGO illustrates visual programming and is used by teenagers and university students to program MindStorm Robots. The fourth stage is the transition to textual programs. To this end we will use environments oriented for the puzzle in the educational programming language MaSH (developed by Dr. A. Rock). MaSH is an imperative subset of the programming language Java (and by removing many of the sophistication of Java, first-year students understand every line of code they use).

Students are provided with guidance and instruction for problem solving (Jones, 1998). We started with classical approaches. An extreme summary (Polya, 1957, www.math.utah.edu/ $\mathrm{pa} / \mathrm{math} /$ polya) has been provided for high-school trials, see Fig. 1. Later, more elaborate instruction on problem solving will be provided. For example, students may be provided with more details on one of the step in Fig. 1. In general, much more sophistication on problem solving will be delivered than can be illustrated here; at each step elaborating on the techniques for problem solving and in particular, for algorithm analysis and design (Skiena, 2008). As alluded to before, activities illustrate a wide range of useful problem-solving skills: analogy, simplification, exploration, iteration, divide and conquer, and recursion. Instruction, illustrations and materials on methods like divide-and-conquer, reduction, and analogy will be formally provided.

\section{ILLUSTRATION}

The activity we use as an example is named "sorting cows" but is based on Sorting Networks (Knuth, 1973, Section 5.3.4) and has similarities with the Sorting Networks activity popularised by "Computer Science Unplugged" (csunplugged.org/sortingnetworks). In its first stage, the participants are introduced to the basic operations of sorting bridges (also named gates). The analogy is a series of rails populated by cows that are connected by the gates. The gates may swap cows across rails, and the generic goal is to design and arrangement of the gates that achieves a certain objective, for example, placing the largest cow in the first rail. These simple operations are comparison operations and swap operations. The Blue (also named large up) operations ensure that the larger cow is in the lower numbered rail when two cows (items) meet at it. The green gate (also named 

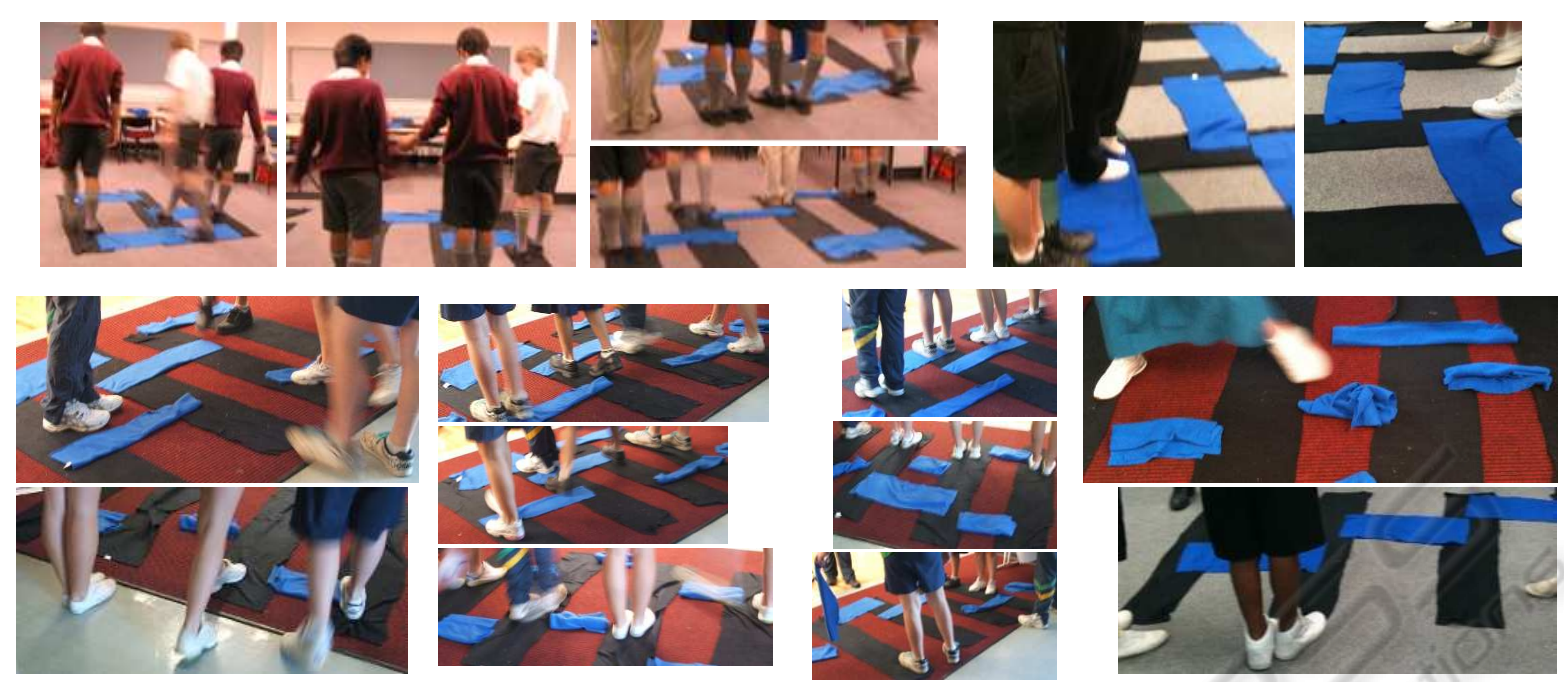

Figure 3: High-schools students constructing sorting networks and experimenting in their own person the effect of their construction.

larger down) always places the larger cow in the larger numbered rail. The red gate always swaps the cows.

\subsection{First Stage - Concrete \& Personal}

In the first stage of the activity, the experience is physical and personal. The participants walk on carpet that represent the rails and experience themselves the fact that they must wait at a gate for another participant in the connected rail, and then perform the required operation as per color (function) of the gate. They experience configurations for 3 or 4 participants and try to discover permutations which fail to reach the objective. For example, the network does not sort. They also may be asked to explore some patterns and to discuss the parameters of the problem. Instruction of problem solving is given by analysing first what problems may be feasible and which may be not. Variations like finding the largest and the smallest without sorting, or using only one type of gates are discussed as well as considering that the cost of bridges/gates may not be always uniform when rails are further apart. Fig. 3 shows high-school students constructing networks and personally navigating them. The images correspond to two Griffith University Experience Days (19th of May and 27th of July 2010), and 3 days (21st, 22nd and 23rd of July 2010) of the Science and Engineering Challenge.

This is what we describe as a concrete experience that is based on Piagetism and will be moved to a more informed and abstract familiarity. The idea is to strengthen the students' semantic schemata (or their building processes) by associating their experience and their action with appropriate language. We expect that such language will include words like sorting, comparison, swap, cost, algorithm, permutation, and the like. It also will have more possibilities for abstractions like the notion of sorting network itself.

Moreover, some inquiry questions that put in practice mathematical problem solving can also be investigated at this stage (participants should be able to argue that in order to sort or to find the largest cow, all rails must connect with at least one gate).

\subsection{Second Stage - Concrete Constructions}

In the second stage of the activity, the participants still work away from computers and continue using concrete objects. In this case, they build with cloth and corresponding strings artefacts that represent the networks. They lay the rails and the bridges and experiment, evaluate and interact their concrete constructions. Fig. 4 shows the types of networks that can be physically built with a white blanket as background, black stripes as rails, and also cloth strings as gates.

We emphasize that the experience remains concrete. The participants are using tangible rails and gates. However, they are able to build larger networks and discuss larger instances. The intention is to initiate the exploration of patterns that may scale to larger solutions. These networks will cause behaviour. We achieve this by capturing them with a camera and executing them in a simulator. The participants construct concrete artefacts that encode behaviour. This is what we name concrete programming.

The realization that this network is a representation of computation (that is, an encoding of be- 

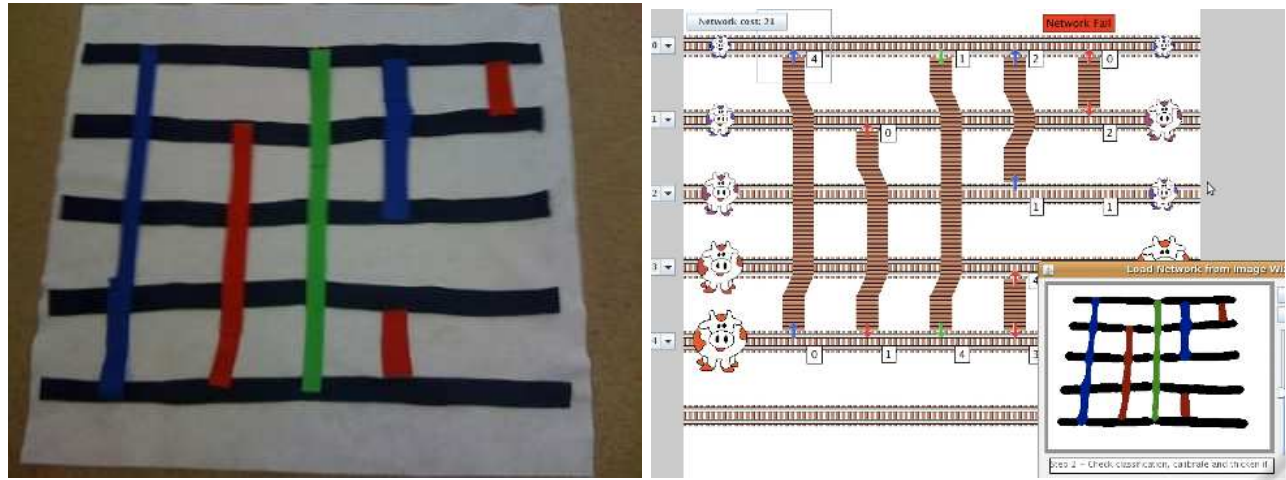

Figure 4: A sorting network constructed of cloth and capturing the network into the virtual environment.

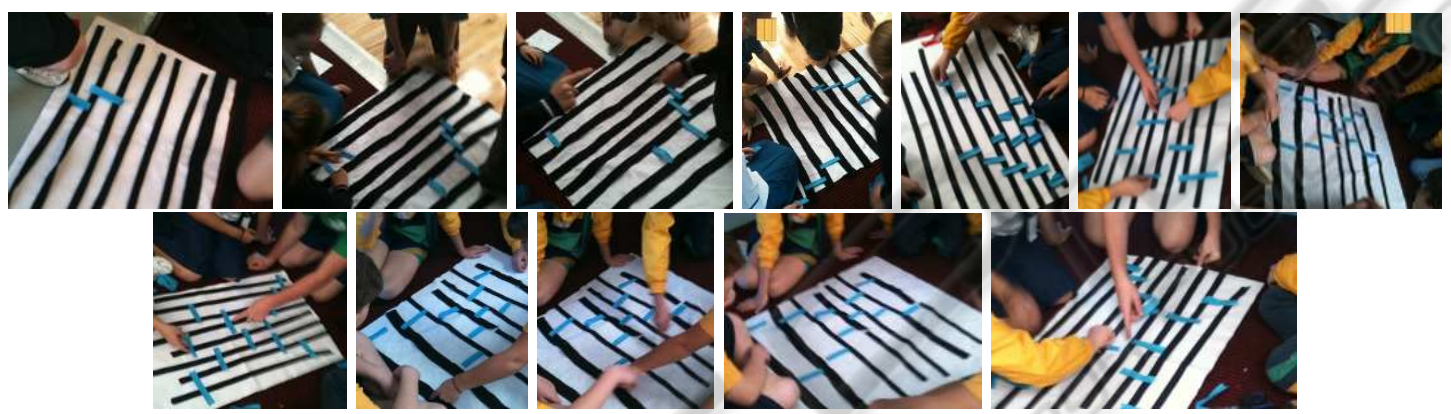

Figure 5: Teams of students working on constructing solutions for selection and sorting problems on networks.

haviour) is achieved by our supporting tools. These networks are captured by a camera (as simple as a mobile phone camera) and fed trough an image recognition software. This image recognition software recuperates the network and can represent it in a graphical user interface that is also coupled with a simulator. The participants can see the effects of the network in any permutation they wish to test it, or on a sample test set or even more in all permutations (as long as the network has less that 9 rails). A participants network can be evaluated against many objectives. The user can chose the objective (like finding the median). They also get feedback on the cost of the network in parameters like gates used, or time used. With this, participants are converting their constructions to a graphical simulation in the computer and observing the effect on their tests.

\subsection{Third Stage - Virtual Constructions}

Once this step is completed, the participants can move completely to the graphical user interface and configure far larger networks that would be possible physically, and explore far more patterns. For this, our earlier software tool allows to interactively add and manipulate all the elements previously experimented physically. Here, participants still manipulate gates, and rails, but on a virtual environment provided by the GUI-enabled tools.

This is the third stage: the experience remains concrete. However, such experience is no longer physical, but is now an immersion into a virtual world. All the actions of the sorting network, and the rails, and the cows belong now in the environment of the simulator and the graphical user interface. The tool provides feedback. It can be tested on specific instances, or on all permutations of 9 or less items. It can sample a large number of permutations for larger instances and also provide feedback on different criteria and cost functions. For example gates that join adjacent rails may be less costly than those that join rails far apart.

Participants can address even larger instance; however, large networks become impossible to build for a particular objective unless one identifies a pattern. The identification of these patterns introduces notions like subproblem. A clear example of this pattern is a common solution to finding the smallest cow and placing it at the bottom rail (see Fig. 6) This pattern enables analysing if the number of blue gates used is optimal for any number of rails. More interestingly, it enables to discuss other aspects of the pattern. If all the gates are replaced by red gates, then the 


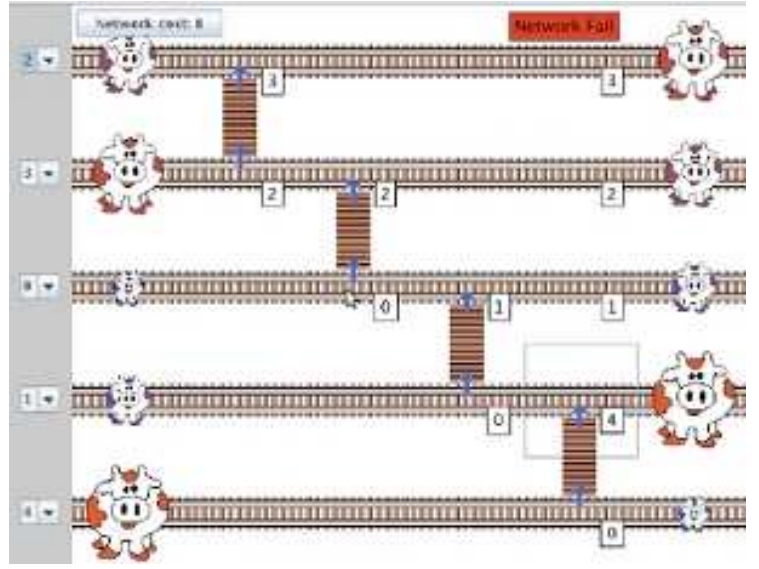

Figure 6: Placing the smallest cow in the bottom rail.

pattern corresponds to a cyclic shift, where each cow moves to the next rail up, except of course the cow on the first rail. The one on the top rail "wraps-up" and appears in the bottom rail.

For example, from repeatedly finding the largest cow with a diagonal pattern, it is possible to scale the pattern down from $n$ rails to $n-1$ rails. The pattern can also be seen as scaling up the basic operator from 2 to 3 rails. However, this pattern can be repeated to create a sorting network by using it repeatedly from size $n$, then $n-1$ and so on down to 2 .

At this stage is also possible to introduce more language. We illustrate the fact that finding the largest item can be used repeatedly for sorting. Moreover, one can discuss many of the sorting networks in the literature and discuss algorithmic strategies. In particular, is possible to introduce algorithmic problemsolving terminology like recursion and divide and conquer without the presentation syntax and semantics of a textual programming language.

We argue that even more important technical concepts can be built. This is what we mean by given the students schemata with strong understanding and meaningful semantics. It is possible to illustrate and argue about the correctness and optimality of the constructions. One can even motivate and introduce the mathematical notion of proof by induction and connect it (Wand, 1980) to the algorithmic strategies.

\subsection{Fourth Stage - Textual Constructions}

From here, the 4-th stage of the activity is the migration to a textual encoding of this behaviour. We introduce this using a particular environment of MaSH (Rock, 2010). MaSH stands for "Making Stuff Happen" and it is a tool to create textual programming

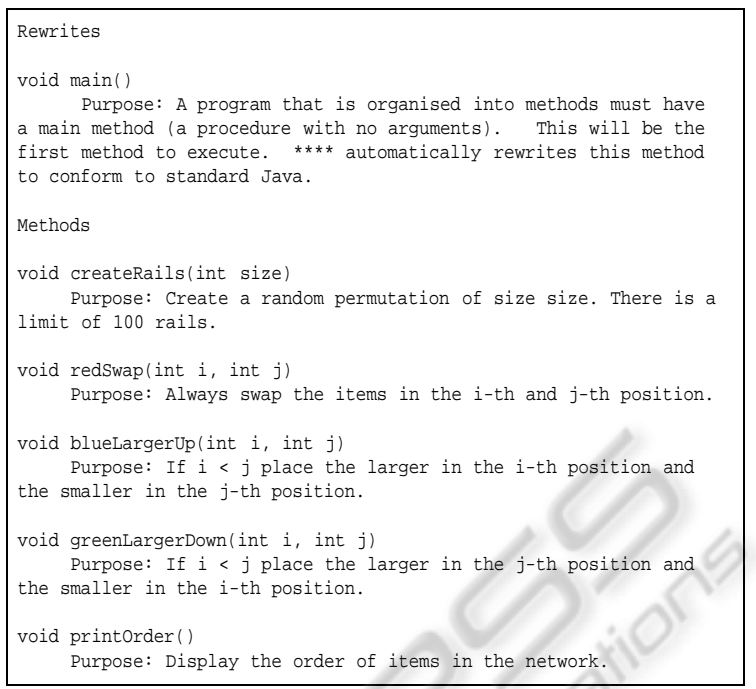

Figure 7: The API of the MaSH environment sortingnetwork.

environments where syntax and semantics are simple. We have constructed one of those environments called sortingnetwork whose documentation appears in Fig. 7. This environment is an API that emulates completely the GUI but enables the introduction of textual programming language concepts. We start with the level named statements in MaSH and can produce a direct mapping between the visual representation of sorting networks in the GUI and MaSHstatement program. For example, the MaSH-statement program in Fig. 8(a) corresponds to a sorting network that has only one gate between two adjacent rails and always swaps the items. The program can be the result of exporting a sorting network built in the virtual environment. It can also be constructed with a text editor (an imported to the tool for visualization). This enables the introduction of concept like "identifier", "separator", and most of the lexical instruments experienced in textual programming languages. Other concepts at the level of statements that the participants can practice and understand are "sequential control", "comments", "displaying output" and "method/function invocation". The simple MaSH program of Fig. 8 is randomised. Each execution generates the values randomly and its output may be as in Fig. 8(b) or as in Fig. 8(c).

There are several sub-levels in this stage of the activity that correspond to the levels in the design of $\mathrm{MaSH}$ but which can be reinforced with the activity. The repetition of the comparator each time in the next rail for all rails results directly into a control structure. That is, we can introduce the notion of a "for-loop". Fig. 9 presents the program where red gates are used to cyclically shift. This program was sufficient for us 


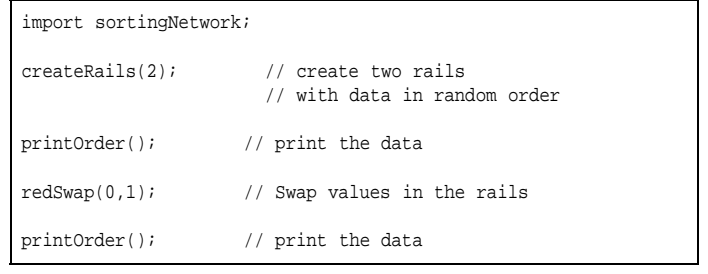

(a) Simple MaSH program at level statements.

\begin{tabular}{|l|}
90,32, \\
32,90, \\
\hline
\end{tabular}

(b) Sample output 1 .

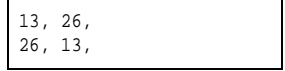

(c) Sample output 2 .
Figure 8: Simple MaSH program and sample output.

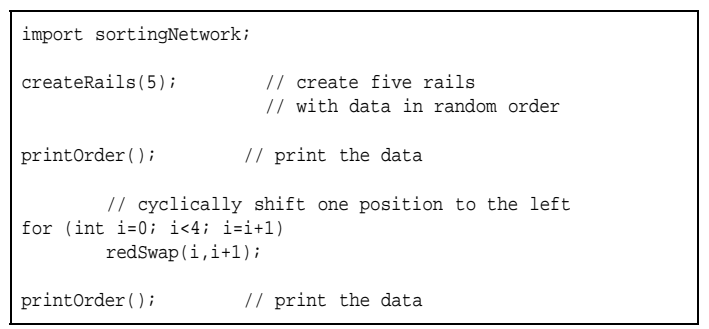

(a) 'For-loop' in a MaSH program at level control structures.

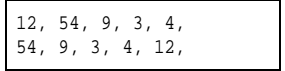

(b) Sample output 1 .

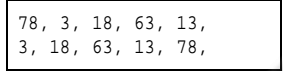

(c) Sample output 2.
Figure 9: MaSH program to introduce control structures.

to introduce control structure concepts to high school students with no programming experience. We could illustrate and have even meaningful discussion about the limit used in the for-loop for the index variable $i$. That is, the program has $i<4$ as the termination condition but the data consists of 5 elements. Significant discussion is generated then about the actual parameters to redSwap in the body of the control structure.

Another aspect that can now be introduced builds on the discussion of patterns from the previous concrete experience. The pattern we described earlier where using a solution to place the smallest as a subproblem to sorting, enables the introduction of the notions of method (or subroutine). For example, we can encode the pattern into a subroutine first. This is illustrated by a MaSH program that corresponds to two invocations of finding the smallest which corresponds to a network to place the two smallest cows in the bottom two rails. The MaSH program corresponding to Fig. 10 is presented in Fig. 11. This enables the introduction of concepts like formal parameters and the control-flow generated by this function invocation. Similarly we can discuss concepts like side-effects although fundamentally all variables are global at this level and there is no data encapsulation. However, the

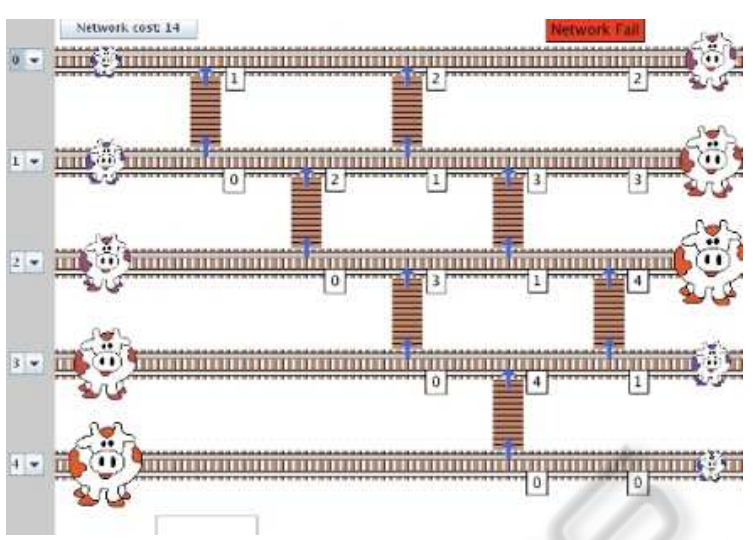

Figure 10: A network that extracts first and second by two applications of the diagonal pattern.

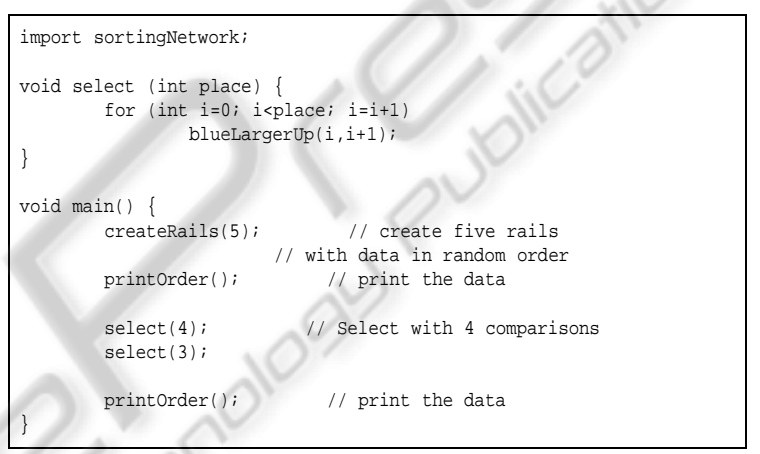

Figure 11: MaSH program using subroutines in the Sorting networks environment.

important aspect is we have a concrete experience to ground the discussion of the abstract elements of textual programming languages. It is important to realize that control structures in the MaSH programming environment become generic in terms of the size of the instance. This is not possible with the concrete sorting networks, which are built for a specific size. This is another point that the participants should observe. It also allows expanding the discussion regarding the generality of a solution in problem solving. The reduction of a problem to another problem for which we already know a solution is a problem-solving strategy that is ubiquitous in algorithm design. We can visualize the repetition of the selection of a smallest element in a sorting network, refer to Fig. 12. The MaSH program of Fig. 11 can be generalised to a sorting program as per Fig. 13. The activity can now progress more rapidly into other programming concepts and in particular, MaSH is designed to introduce behaviour first and later introduce data, and finally introduce the plethora of object-oriented concepts. 


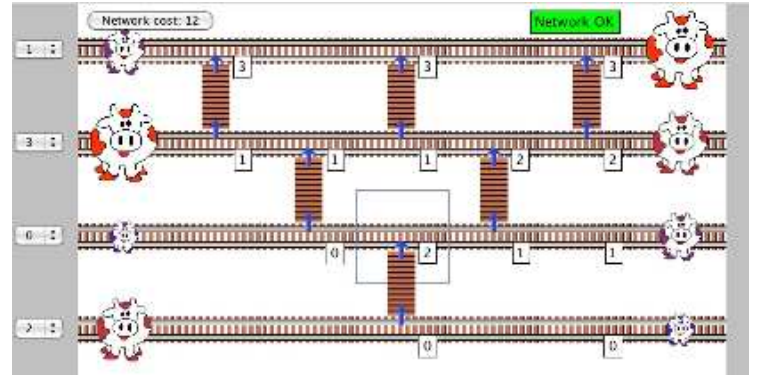

Figure 12: A sorting network.

\subsection{Stage Five - Advanced Topics and Research}

We envisage also a later stages of these activities, that can lead to artificial intelligence (like to search for cost optimal networks for specific parameters of the problem), or even to research challenges, like to establish the best network for certain cost structures and sets of operators. The activity also leads itself to consider concurrency and parallelism and the introduction of programming concepts in this area. In particular, the tools has been used in ICT2501 Programming 3 to illustrate the algorithmic complexity of selection sort. The tool provides a visual tangible (concrete) illustration that selection sort requires $O\left(n^{s}\right)$ comparisons, since the sorting network uses gates in all positions on one half of a grid of width $n$ and thus, of area $n^{2}$.

\section{THE EXPERIENCE}

We have performed these two activities with 14 groups of participants from high-schools as part of the events mentioned earlier (a total of 56 students organised in groups and additionally 8 students as individuals). These were conducted as trials for the activities. Each activity was conducted at least once by a person who was not the designer of the activity, who is not an academic and not trained as an instructor but who had witnessed the activity once. There were no major differences in the execution of the activity and all others who were conducted by Estivill-Castro (one day with assistance of Dr. S. Venema). Our intention was to evaluate the following aspects.

1. Do the participants acquire technical language and have a have better semantic understanding for the concepts of such language?

2. Do the participants acquire and understanding that problem-solving is a strategic and acquired skill?
3. Does the concrete experience assist in problemsolving?

4. Is the organization in teams useful?

\subsection{Semantic Understanding of Language}

The first research issue was evaluated by a short interview before the activity and a questionnaire after the activity. The interview assessed the previous understanding of some terminology while the questionnaire investigated the understanding of activity related technical concepts and the use of new terminology. The results were promising. For example, prior to the activity, very few of the students could enunciate a clear definition of the notion of median and even less clear was any connection with the notion of mean. However, by the end of the activity, the notion of median was clearly described by the participants and they could make the observation themselves that it is not uniquely defined when there is an even number of items. Some participants were capable of realizing that the median is a robust estimator of the central tendency and that enlarging the largest item or reducing the smaller element does not affect the value of the median (however, such changes in the data do affect the mean).

There was a similar visible extension on the understanding of terms like algorithm, problem, instance of a problem, verification, and correctness. Many other concepts were not formally evaluated by the questionnaires but it was clear the participants gained insight. For example, they understood that testing all permutation to verify correctness of a sorting network grows very rapidly with the number of rails (items). Similarly, some realised that verifying the network is the most economical (by a criteria like least number of gates) was even more unfeasible to verify by exhaustive exploration. Other terminology like cyclic shift, coding and programming language also showed improvements.

In fact, some students were able to make very insightful observations. That is, to enunciate a claim and then provide and argument for justifying its validly. This emulated the formulation of a theorem and its proof although not structured in this way. An example of this happening is "In a network with only blue gates, the smallest element never finishes in a rail above to where it started". We find interesting that from this, the team of participants where it emerged was able to rapidly generalize how to find the largest item, and/or the smallest, and then discover patterns and build a sorting network that would solve problems for all sizes. They were also able to structure ar- 


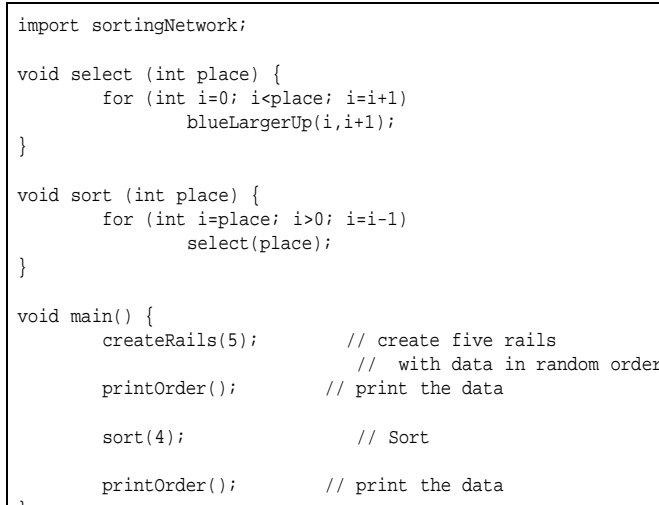

Figure 13: MaSH program using subroutines for sorting.

guments for the correctness of their approach. Moreover, they could then formulate other claims about the need for red or green gates in order to place the smallest element above the rail it starts (in particular, they could made the observation about the impossibility of sorting in descending order only with blue gates).

\subsection{Strategies for Problem Solving}

For this we also had a questionnaire after the activity asking if the information on problem solving was useful (information along the lines of Fig. 1 in one A4 page was distributed to participants). We also had one control group to whom no instruction on problem solving was provided neither any material on problem solving .

In this case the informal observations we performed offered some contradictory observations. Some participants did not seem to be able to attack the problems in any way, particularly those that were not in groups. Some high-school students seemed preoccupied with there being an answer they should have previous knowledge for and seem to see the challenges of the activities as test on their memory. However, the vast majority engaged in the activity, particularly as we mentioned, when grouped in teams.

The positive outcomes in this regard were many. For example, some participants were able to apply effectively strategies like "start with a smaller problem" and they used the solution from finding the largest item to find a network to sort. Several could indeed examine the solution they obtained and generalize it. Some were able to reflect on the problem statement and discuss their understanding of the challenge. They could be systematic and eliminate possibilities or consider all cases. They could understand what a counter-example means.

We also found some very genuine ideas. For ex- ample, we found that two groups actually found a very interesting and to our knowledge innovative approach to construct a sorting network. Because the first problem provided to them in the first phase is to find the smallest item on 3 rails, teams commonly produced a diagonal optimal pattern like in Fig. 6. (see bottom right image on Fig. 3). The next problem was to find the maximum. For this problem, some teams used the previous idea but now the diagonal pattern follows placing the next blue gate on the winner and runs symmetrically in the other direction (see Fig. 14(a)). It is not hard to compose these two patterns to identify the smallest and the largest items in a network with four rails (see Fig. 14(b) and Fig. 14(c)). When the students attempted to sort on a network of 4 rails they realised that they only need to ensure the two rails in the middle work. This produces the sorting network shown in Fig. 14(d) and visible in the images on the bottom left of Fig. 3. This network is extremely efficient for sorting 4 rails, requiring only 3 time steps (better than the pyramid pattern in Fig. 12). What we found extremely interesting is that when this approach is generalised to 8 rails in the constructions with tape, some of the teams produce the network that reduces the problem of sorting $n$ rails to sorting the $n-2$ middle rails and produce a network like in Fig 14(e). Besides a visually appealing pattern, this network has some interesting properties, it uses only blue gates besides adjacent rails (no long gates) and it uses the same number as the corresponding pyramid pattern of Fig. 12. While it requires more parallel time units, it does have some degree of parallelism.

Although we have no space here to describe the Dinosaur activity suffice to say it consists of describing heuristic algorithms for the NP-Complete problem VERTEX COVER ${ }^{3}$. Participants spontaneously produced randomised heuristics, algorithms based on local search (selecting all vertices and then removing unnecessary vertices from the cover plus local perturbations), algorithms based on structure finding (vertices of degree one, or triangles - cycles of length 3 ) and greedy algorithms (choosing a vertex of largest degree). The notion of a competitive algorithm did not emerge as this seems an advanced concept.

\subsection{Usefulness of the Concrete Experience}

We were interested in investigating if the first stage of the activity (the concrete experience on the participant) has a positive impact. Thus, we reserved 8 par-

\footnotetext{
${ }^{3}$ To cover edges by selecting the minimum number of vertices in a graph. An edge is covered if at least one of its endpoints is in the selected cover.
} 


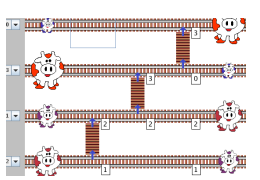

(a) Largest of 4 rails.

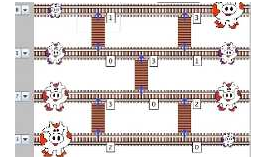

(b) Optimal $\mathrm{x}$ paralle pattern to find largest and smallest item in for rails.

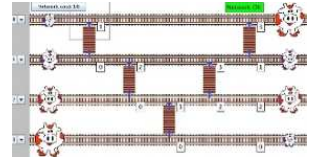

(c) Sequential $\mathrm{V}$ pattern to find largest and smallest.

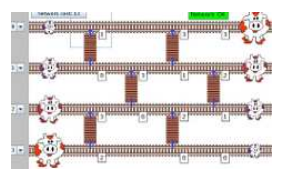

(d) A gate in the middle rails sorts.

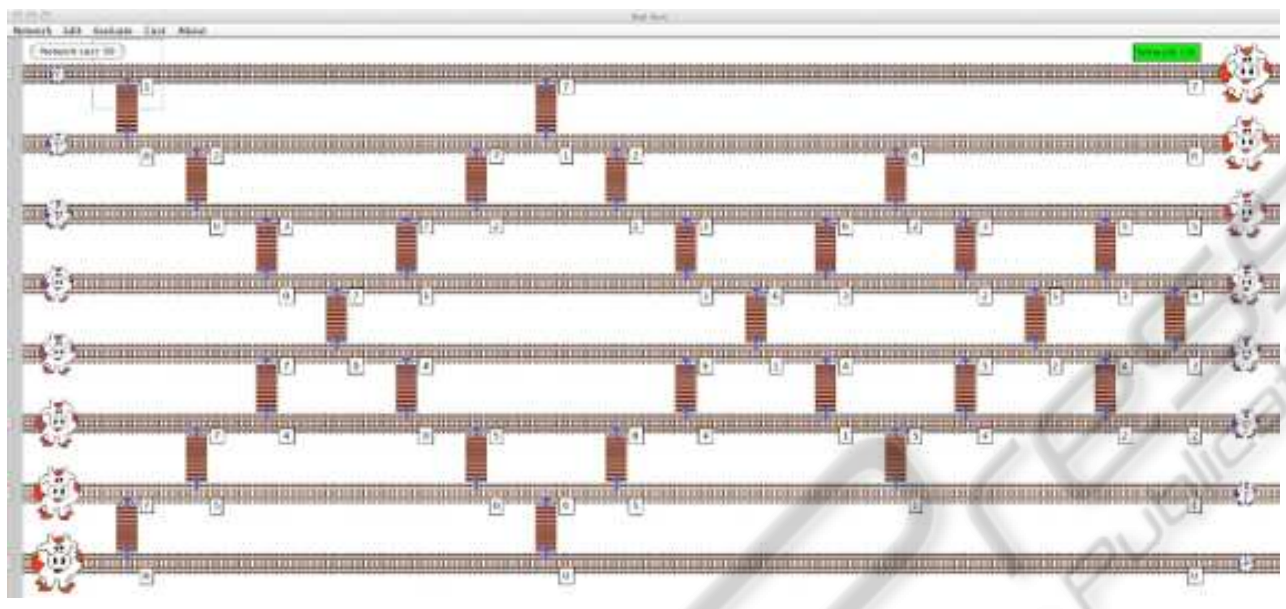

(e) Sorting network from recursive application of X pattern.

Figure 14: A very symmetrical sorting network by a recursion that finds simultaneously the largest and the smallest item.

ticipants who were not involved in the first phase an their involvement commenced with the second phase where the construction is concrete but not personal. We used two measures to evaluate the difference between these 8 participants and the others. First, we measured the number of times they required further assistance or clarification by the person conducting the activity. Second, we measured the time it took for them to complete activities of the second phase as well as the time it took them to complete problems using the virtual rails tool (stage 3 ).

The second measure was the number of times there was a request for assistance or clarification from the activity conductor or the assistant. While the data may have a significant margin of error in measurement it does reflect that high-school students that participated in a physical phase of the activity where each person had to role-play a cow (or a dinosaur) eventually was getting better at facing the new problems and the new challenges. Those that skip the role-play phase save the time of this part and thus, complete the challenges of the concrete part (phase 2) perhaps sooner or at least not worse than those that did the role-playing. However, these second group of participants struggle with the later challenges and activities. For example, we proposed to find the median element and place it in a specific position using the virtual tool on a network of 9 rails, very few of the participants without the role-playing could complete this activity. assistant(s). Fig. 15 shows the comparisons in phase 2 challenges (build a network to find the maximum, find a network to find the minimum, find a network that sorts) and one challenge from phase 3 (place the largest on the top rail and the smallest in the second rail). The data reflects the trend that individual work is not as effective as during the different activities the average number of queries remains above 1 per student, suggesting is more efficient to group the participants in teams as this seems they assist each other.

\subsection{Organization in Teams}

We measure the same two aspects of participants in teams and compared against 8 participants that were not placed in teams. The data reflects that teams progress better with the later challenges than those that work in isolation. However, we are not sure that everyone in the team reaches the same command of the concepts and ideas.

Team-work proved to be productive and we can feel confident that the teams progress well. One interesting aspect is that we were able to observe actual collaboration and participation. It is usually difficult to see this type of interaction between members of a team in traditional laboratories for delivering programming concepts or problem solving concepts. In 

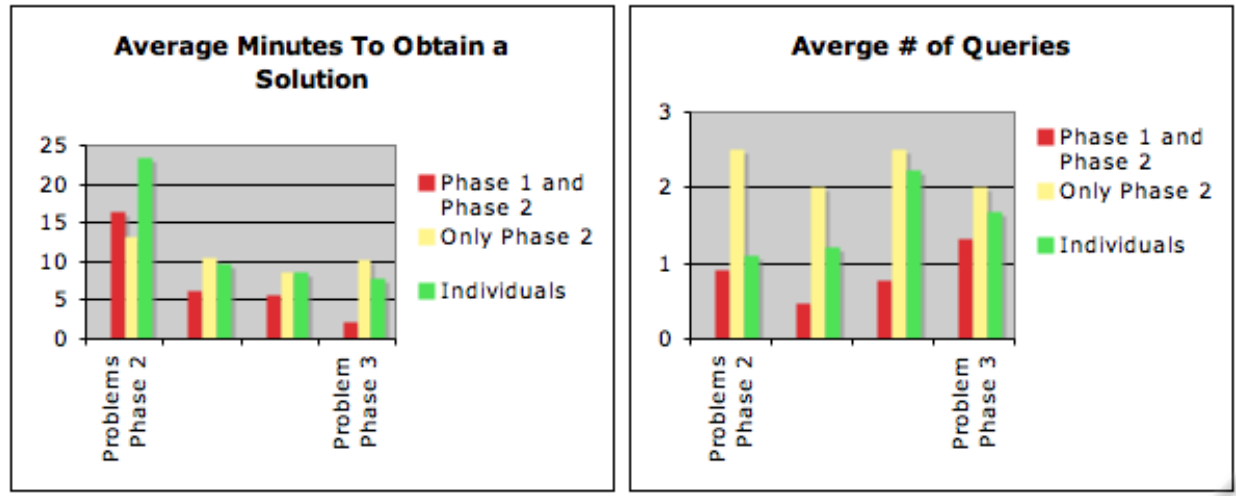

Figure 15: Comparing students in groups, as individuals and groups without phase 1 in completing a network for 4 types of progressively more advanced problems.
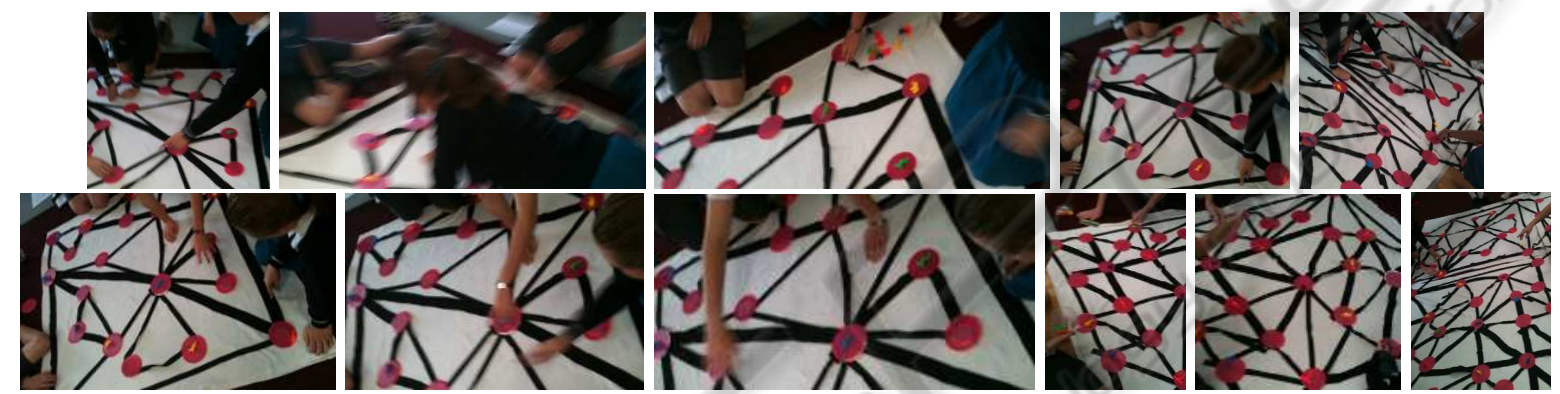

Figure 16: Teams of students working on constructing difficult instances of vertex cover (cities) and then actual covers (dinosaurs on vertices guarding edges).

particular, with current labs suited with workstations fitted for individual use it is very rare to see two students collaborating and having meaningful discussion with the physical limitation of one monitor. We can see that we were able to produce constructive interactions with all team members as can be seen in the images of Fig. 5 and Fig. 16. The images reveal several situation in with a a hand from at least 3 participants, if not all four in a team, actively engaging with the concrete construction. This suggests complementing teaching labs with interactive whiteboards or other technologies where students could interact in groups and collaborative edit and modify the current design or solution. Thus, we see an earlier introduction to Computer Supported Cooperative Work.

\section{CONCLUSIONS}

This paper present initial approaches to teach problem solving for programming as the construction of physical artefacts (and not textual items). While visual programming enables concrete manipulation of objects, the environment is still virtual. We propose to commence with the personal and physical experi- ence as a preliminary step and use technology to capture images and video of such constructs in order to produce a behaviour in a system.

\section{ACKNOWLEDGEMENTS}

This work would not have been possible without the programming talent and skill of Nathan Lovell $\mathrm{PhD}$, and the financial support of a Teaching and Learning Grant from Griffith University.

\section{REFERENCES}

Adams, J., Kaczmarczyk, S., Picton, P., and Demian, P. (2007). Improving problem solving and encouraging creativity in engineering undergraduates. In International Conference on Engineering Education ICEE07, Coimbra, Portugal.

Adams, J. and Turner, S. (2008). Problem solving and creativity for undergraduate engineers: process or product? Innovation, Good Practice and Research in Engineering Education, page 61.

Biggs, J. (1999). Teaching for Quality Learning at University. Shire and Open University Press, UK. 
Brown, S. and Walter, M. I. (1970). What if not? an elaboration and second illustration. Mathematical Teaching, 51:9-17.

Brown, S. and Walter, M. I. (1990). The art of problem posing. Lawrence Earlbaum, Hillsdale, NJ, second edition.

Cindy E. Hmelo-Silver, C., Duncan, R. G., and Chinn, C. A. (2007). Scaffolding and achievement in problembased and inquiry learning: A response to Kirschner, Sweller, and Clark (2006). Educational Psychologist, 4(2):99-107.

Dale, N. and Weems, C. (2002). Programming and Problem Solving with $C++$. Jones and Bartlett Publishers, Inc., USA, 3rd edition.

Dewar, J. M. (2006). Increasing maths majors' success and confidence through problem solving and writing. In Rosamond, F. and Copes, L., editors, The Influences of Steven I, Brown, pages 117-121, Bloomington, Indiana. Educational Transformations, AuthorHouse.

Estivill-Castro, V. (2010). Concrete programing for problem solving skills. In Gómez Chova, L., Martí Belanguer, D., and Candel Torres, I., editors, International Conference on Education and New Learning Technologies (EDULEARN 2010), pages 4189-4197, Barcelona, Spain. International Association of Technology, Education and Development (IATED). CDROM file 454.pdf, ISBN: 978-84-613-9386-2.

Etter, D. M. and Ingber, J. (1999). Engineering Problem Solving with C. Prentice-Hall, Inc., Englewood Cliffs, NJ.

Houghton, W. (2004). Learning and Teaching Theory for Engineering Academics. Engineering Subject Centre.

Jones, M. (1998). The Thinker's Toolkit: 14 Powerful Techniques for Problem Solving. Three Rivers Press, USA.

Knuth, D. (1973). The Art of Computer Programming, Vol.3: Sorting and Searching. Addison-Wesley Publishing Co., Reading, MA.

Koffman, E. (1988). Problem Solving and Structured Programming in Modula-2. Addison-Wesley Publishing Co., Reading, MA.

Michalewicz, Z. and Michalewicz, M. (2008). PuzzleBased Learning - An Introduction to Critical Thinking, Mathematics, and Problem Solving. Hybrid Publishers Pty Ltd, Victoria, Australia.

Pask, G. (1975). Conversation, cognition and learning. Elsevier, New York.

Polya, G. (1957). How to Solve It: A New Aspect of Mathematical Method. Princeton University Press, second edition.

Prince, M. and Hoyt, B. (2002). Helping students make the transition from novice to expert problem-solvers. In 32 Annual Frontiers in education (FIE-02), volume 3, pages F2A7-11, Los Alamitos, CA, USA. IEEE Computer Society.

Rock, A. (2010). Creating MaSH programming environments. School of ICT, Griffith University.

Savitch, W. (2009). Problem Solving with $C++$. AddisonWesley Publishing Co., Reading, MA, 7th edition.
Schneider, M. (1982). An introduction to programming and Problem Solving with Pascal. Addison-Wesley Publishing Co., Reading, MA, 4th edition.

Skiena, S. S. (2008). The Algorithm Design Manual. Springer-Verlag, London, second edition.

Toussaint, G. (1993a). A new look at Euclid's second proposition. The Mathematical Intelligencer, 15(3):12-23.

Toussaint, G. (1993b). Un nuevo vistazo a la segunda proposicion de Euclides. Mathesis, 9:265-294.

Vygotsky, L. S. (1978). Mind and society: The development of higher psychological processes. Harvard University Press, Cambridge, MA.

Wand, M. (1980). Induction, Recursion and Programming. Elsevier Science Inc., New York, NY, USA.

Wickelgren, W. (1995). How to Solve Mathematical Problems. Dover, New York.

Woods, D., Wright, J., Hoffman, T., Swartman, R., and Doig, I. (1975). Teaching problem-solving skills. Engineering Education, 66(3):238-243. 